\title{
Medicina de Emergencias y Desastres: los últimos 50 años
}

\section{Emergency and Disaster Medicine: the last 50 years}

\section{Nelson Raúl Morales-Soto ${ }^{1}$}

1. Academia Nacional de Medicina,

Sociedad Peruana de Medicina de Emergencias y Desastres, Universidad Nacional Mayor de San Marcos.
Todos los pueblos tuvieron capacidad para dar algún tipo de atención a enfermos y heridos, la historia de la Medicina de Emergencias y Desastres ${ }^{1}$ se ha construido por la alternancia de eventos adversos (grandes emergencias, desastres) y de hechos positivos como la creación de instituciones, innovaciones y contribuciones (descubrimientos, implementación e institucionalización de organizaciones. normas v otros recursos para la protección y asistencia en caso de eventos adversos), por lo común los primeros sirvieron de catalizador y alentaron o aceleraron la implementación de los segundos. ${ }^{2}$

Un evento adverso ocurrido en el Estadio Nacional de Lima hace medio siglo, el 26 de mayo de 1964, ocasionó la muerte de 328 personas y lesiones a otras mil. Nos enseñó que las normas de seguridad para las grandes concentraciones humanas tenían que ser más estrictas y que los establecimientos de salud debían disponer de procedimientos para la atención de demanda masiva de víctimas graves.

Con esta necesidad se preparó un plan hospitalario para desastres en 1965 en el Hospital Central de Policía el cual fue probado y corregido en los sismos de 1966, 1970 y 1974, los procedimientos operativos del mismo fueron publicados en $1983 .{ }^{3}$

El terremoto del 31 de mayo de 1970-con magnitud de 7,8 grados Richter y epicentro marino frente a Áncash-fue seguido de un aluvión en el Callejón de Huaylas causando la muerte a 65000 personas y heridas a otras 160000; ha sido catalogado como el terremoto más mortífero de ese siglo en el continente americano; la respuesta nacional e internacional fue masiva, en junio de ese año el doctor Donald Morote organizó el Hospital de Campaña del Instituto Peruano de Seguridad Social para atender a las víctimas. ${ }^{4}$

Este sismo mostró las proporciones descomunales que podía alcanzar una catástrofe y desnudó la falta de preparación reinante en el país, esto dio origen a la creación del Instituto Nacional de Defensa Civil, Decreto Ley N. ${ }^{\circ} 19338$ del 28 de marzo de $1972^{5}$ y se iniciaron masivamente los preparativos hospitalarios para desastres.

El 25 de febrero de 1912 fue inaugurada la Asistencia Pública de Lima, en 1956 ya disponía de 6 puestos de socorro; 36 médicos organizados en 3 equipos llegaban a atender hasta 500 pacientes en los días comunes y 1500 en domingos y feriados. El Puesto Central de la avenida Grau fue cerrado en julio de 1980 cuando se inaugura el Hospital de Emergencias 'José Casimiro Ulloa', 6 el cual contaba con 4 quirófanos, 100 camas de internamiento, unidad de Cuidados Intensivos, exámenes auxiliares y tecnología adecuada a su tiempo. Con el tiempo se sumaron especialistas de otros campos para completar los equipos de guardia y asegurar una atención calificada.?

En 1980 se iniciaron las actividades terroristas del movimiento subversivo 'Partido ComunistaSendero Luminoso'. En 1981 ocurrieron 700 atentados y asesinatos de policías, la actividad destructiva fue en aumento, en 1992 se produjo un atentado con $500 \mathrm{~kg}$ de explosivos en la calle Tarata, en Miraflores, que dejó 23 muertos y 132 heridos (64 de ellos graves). El 25 de mayo de 2001, 
el gobierno aprobó la creación de una Comisión de la Verdad y Reconciliación (CVR) para analizar el fenómeno subversivo y sus efectos y formular un programa de reparaciones; en su informe establece que la cifra de víctimas entre muertos y desaparecidos llegó a 69280 personas, $40 \%$ de ellos se concentraron en Ayacucho, $79 \%$ fueron campesinos y $75 \%$ tenían el quechua como idioma materno. ${ }^{8}$

Las cifras de atención en Emergencia, al concluir el año 1982, habían duplicado las de 1978 pero la incidencia de graves y demanda masiva de víctimas de explosiones había crecido exponencialmente, generando $55 \%$ de los internamientos nosocomiales. En 1983, el Hospital de Policía decidió crear una unidad especializada para atención de emergencias graves: unidad de Shock-Trauma, en ella se inicia, ese mismo año, el triaje en Emergencia, el transporte asistido en ambulancias con radioenlace, la cirugía de reparación de daños; las etapas del proceso fueron publicadas y el modelo se extendió ${ }^{9-11}$ y permitió la creación de la especialidad en Medicina de Emergencias y Desastres en la Facultad de Medicina San Fernando, Universidad Nacional Mayor de San Marcos, el 10 de setiembre de 1992 (Acuerdo N. ${ }^{\circ} 468$ del Consejo de Facultad tomado en su sesión XXVIII).

En mayo de 1982, los jefes y médicos de los servicios de Emergencia de los hospitales de Lima definieron su interés en formalizar una sociedad médica especializada y dan nacimiento a la actual Sociedad Peruana de Medicina de Emergencias y Desastres.

En 1982-1983 se produjo en el país el fenómeno El Niño en categoría calificada como 'muy intenso', con efectos catastróficos en 16 departamentos, por lluvias torrenciales, desborde de ríos, inundaciones, huaicos y sequías (particularmente graves en el altiplano sur). Los departamentos más afectados fueron los de Piura y Puno. Se registraron 1267720 afectados, 512 muertos, 1304 heridos y 25100 enfermos, 587120 personas quedaron sin vivienda. Se afectaron 111000 viviendas y se destruyeron otras 98000 . Se exacerbaron la malaria y la peste y paralelamente se afectaron 260 establecimientos de salud. Se calcularon las pérdidas en 1000 millones de dólares y se afectó el PBI en $-12 \%$. En
1997-1998 se repitió el evento con características igualmente destructivas, las pérdidas económicas se estimaron en 1800 millones de dólares (1 200 millones en bienes públicos). ${ }^{12}$

En 1985, por D.S. N. ${ }^{\circ}$ 057-89-SA, el Ministerio de Salud creó la Unidad de Preparación contra Desastres (UPCD), como un servicio dependiente de la Alta Dirección y con alcance nacional. En 1986, aprueba su manual de Organización y Funciones por R.M. N. ${ }^{\circ}$ 0069-86-SA/DVM, en la que se la define como un órgano normativo, asesor y supervisor, y se le encarga la planificación, coordinación y ejecución de las acciones de salud necesarias para afrontar las situaciones de desastre e intervenir en aquellos fenómenos que amenazaran la vida o la salud de la población y excedieran la capacidad de respuesta de la comunidad afectada. El 28 de abril de 1987, por D.S. N. ${ }^{\circ}$ 022-87-SA, la unidad pasa a depender de la Oficina de Defensa Nacional (ODN) del Minsa, organismo integrante de la Alta Dirección, cuya función principal era conducir la formulación del Plan Sectorial de Acción contra Desastres y Emergencias, en labor coordinada con los gobiernos locales y regionales. En el año 2001, las funciones de la ODN fueron incorporadas al Reglamento de la Organización y Funciones del Ministerio de Salud a través de la R.M. N. ${ }^{\circ} 410-$ 2001-SA/DM. ${ }^{13}$

En 1986, la R.M. N. ${ }^{\circ}$ 597-86-SA/DM designó la Comisión Permanente del Sistema de Atención de Emergencias de Lima-Callao, con la finalidad de diseñar la estructura organizativa del sistema, cuyas bases preliminares habían sido diseñadas en el Plan Operativo para las Atenciones de Emergencia Médico-Quirúrgica en la VI Región de Salud de Lima, propuesto por la Comisión designada por R.V.M. 0026-82 y publicado en 1983. El intenso trabajo de la comisión se volcó en su informe final el cual fue publicado en 1988. ${ }^{14}$

Las condiciones de saneamiento ambiental fueron propicias para que en la última semana de enero de 1991 se presentaran los primeros casos de cólera en el barrio La Candelaria, en Chancay. Casi en simultáneo se registraron casos en Chimbote, en sus tres hospitales se atendieron 900 pacientes en la primera semana y unos 300 diarios durante el mes de febrero, en que la enfermedad se extendió a la costa y la sierra, El cólera llegó a la selva en 
marzo. Al finalizar el año se reportaron 322 mil enfermos y unos 3 mil fallecidos. ${ }^{15}$

En 1997, el Centro Peruano Japonés de Investigaciones Sísmicas y Mitigación de Desastres (Cismid) de la Universidad Nacional de Ingeniería (UNI) desarrolló estudios de vulnerabilidad sísmica en 14 grandes hospitales del país los cuales mostraron un importante grado de vulnerabilidad en establecimientos públicos. Estos estudios fueron ratificados en el año 2013, por la misma entidad con similares resultados.

El 29 de diciembre de 2001, la demostración de un artefacto pirotécnico desató un incendio que alcanzó grandes proporciones en el centro de Lima comprometiendo unas cinco cuadras de galerías comerciales en Mesa Redonda, en las cuales, además de la extendida tugurización, hacinamiento y sobreocupación de mercadería se almacenaban unas 900 toneladas de pirotécnicos. El evento causó la muerte a 289 personas, heridas a otras 600 (gran parte de ellas graves), 352 desaparecidos y pérdidas materiales por unos diez millones de dólares. ${ }^{16}$

Hoy se sabe que los desastres tienen un carácter cíclico, algunos por la constancia de las amenazas, otras por el crecimiento de las vulnerabilidades. En los últimos 50 años se ha aprendido y avanzado mucho pero, en opinión de expertos, no es suficiente para encarar un terremoto destructor al cual, históricamente, se avecina el litoral central. El Sistema Nacional de Gestión del Riesgo de Desastres (Sinagerd) deberá fortalecer los organismos, mecanismos y recursos necesarios para desarrollar una efectiva gestión del riesgo y preparar las ciudades para enfrentar los efectos de eventos catastróficos.

\section{REFERENCIAS BIBLIOGRÁFICAS}

I. Lastres, JB. Historia de la Medicina Peruana. Volumen I: La Medicina Incaica. En: Eguiguren L. Historia de la Universidad, Publicaciones del Cuarto Centenario de la Universidad Nacional Mayor de San Marcos. Lima: Santa María; I95I.

2. Morales-Soto NR. Preparación para emergencias y desastres. En: Academia Peruana de Salud, editor. Historia de la Salud en el Perú. Lima:APS; 2008.

3. Morales-Soto NR. Proyecto de Manual de Procedimientos Operativos en Desastre. Rev San Fuerzas Polic. 1983;44:185198.

4. Morales-Landeo E. Biografía de Donald Morote Revolledo, Historia del servicio de Neurocirugía I3-B, del Hospital de Campaña del IPSS y de la Unidad de Neurocirugía PediátricaUNCIP del Hospital Nacional E. Rebagliati Martins. Lima; HNERM; 2008.

5. Instituto Nacional de Defensa Civil - Indeci [Internet]. Lima: Indeci [actualizado 10 jul 20I3; citado 10 jul 20I3]; [aprox. 2 pantallas]. Disponible en: http://www.indeci.gob.pe

6. Guillén J. Preparación para emergencias y desastres: La asistencia pública, avenida Grau: inauguración. En: Academia Peruana de Salud, editor. Historia de la Salud en el Perú. Lima:APS; 2008.

7. Untama J. Preparación para emergencias y desastres: Hospital de Emergencias “José Casimiro Ulloa”. En:Academia Peruana de Salud, editor. Historia de la Salud en el Perú. Lima:APS; 2008.

8. Comisión de la Verdad y Reconciliación (Perú) - CVR. Hatun Willakuy:Versión abreviada del Informe Final de la Comisión de la Verdad y Reconciliación. Lima: Navarrete; 2004.
9. Morales-Soto NR. Organización de emergencias y formación de emergencistas. Diagnóstico. 1984; I3:2। 3-216.

10. Morales-Soto N, Pinamonti F, Ríos J, Vigo J, Acosta C. Espectro de morbilidad en emergencia. Diagnóstico. 1984;14:73-76. Disponible en: http://www.cridlac.org/digitalizacion/pdf/spa/ doc2778/doc2778-contenido.pdf.

II. Morales-Soto NR. Médicos emergencistas y unidades de shocktrauma. Bol Soc Per Med Emerg. 1985;1:9-II. Disponible en: http://cidbimena.desastres.hn/docum/crid/Julio-Agosto2005/ CDI/pdf/spa/doc3065/doc3065-contenido.pdf

12. Instituto Nacional de Defensa Civil - Indeci; Dirección de Estadística - DNO. Compendio estadístico de emergencias producidas en el Perú 1998. Lima: Indeci; 1998. Disponible en: http://www.indeci.gob.pe/compend estad/I998/I present.pdf

13. Ministerio de Salud/Oficina General de Defensa Nacional/Oficina Ejecutiva de Estudios Estratégicos y Doctrina. Nacimiento y creación de la Oficina General de Defensa Nacional. Lima: Minsa; 2006.

14. República del Perú. Ministerio de Salud. Resolución Ministerial $N^{\circ}$ 597-86-SA/DM, de 1986, que designa la Comisión Permanente del Sistema de Atención de Emergencias de Lima-Callao.

15. Maguiña C, Santana J. Preparación para emergencias y desastres: Epidemia de cólera. En: Academia Peruana de Salud, editor. Historia de la Salud en el Perú. Lima:APS; 2008.

16. Arce Palomino JL. Preparación para emergencias y desastres: Incendio en Mesa Redonda. En: Academia Peruana de Salud, editor. Historia de la Salud en el Perú. Lima:APS; 2008.

\section{Correspondencia}

Dr. Nelson Raúl Morales-Soto moralessotonelson@gmail.com 